\title{
Über das Problem der Freiheit auf Grund von Kants Kategorienlehre.
}

\author{
Von \\ Dr. J. Stilling, \\ Professor an der Universität Straßburg. \\ Erstes Kapitel. \\ Die Aufgabe. ${ }^{1}$ )
}

Wie bei jeder erkenntnistheoretischen Untersuchung handelt es sich auch bei der über die Freiheit des Willens um die genaue Definition der Begriffe. Was heißt Freiheit? Es gibt der Sprache nach sehr verschiedene Arten von Freiheit, es gibt Schmerzfreiheit, politische Freiheit, Freiheit des Denkens, des Handelns, moralische Freiheit. Gleichwohl fallen alle diese Begriffe unter den Allgemeinbegriff der Freiheit, der rein formal erklärt werden muß, während die Einzelbegriffe einen bestimmten materialen Inhalt aufweisen.

Um einen Begriff genau zu definieren, gibt es keinen andern Weg, als der von Kant in der Lehre von den Kategorien vorgezeichnet worden ist.

Unsere gesamte Erfahrung, sei sie einfach der sprachliche Ausdruck von Gefühlen und Anschauungen, sei sie wirkliche Wissenschaft, drückt sich in Begriffen aus. Sei deren Anzahl noch so groß, so muß sie sich dennoch auf eine bestimmte Anzahl von nicht weiter zerlegbaren Grundbegriffen reduzieren lassen. Aus den transzendenten Vermögen der Psyche, diese Begriffe zu bilden, besteht eben unsere Vernunft. Eine solche seelische Macht nennt

1) Die philosophische Grundlage dieses Kapitels findet der Leser ausführlicher dargestellt in meiner "Psychologie der Gesichtsvorstellung nach Kants Theorie der Erfahrung“, (Berlin und Wien 1901 Urban \& Schwarzenberg.) 
man Kategorie, den Begriff, der sie ausdrückt (in dem sie erscheint), einen kategorialen Begriff. Doch gebraucht man gewöhnlich den Ausdruck "Kategorie" für den Begriff, statt daß man damit eigentlich die seelische Funktion bezeichnea sollte.

Diejenigen Begriffe, welche die Arten unserer Sinnlichkeiten ausdrücken (also licht, Ton, Gefühl usw.), sowie endlich diejenigen Begriffe, welche die Formen der Anschauung sprachlich bezeichnen (also Raum, Zeit, Empfindung, Bewegung), sind natürlich nicht zu den kategorialen Begriffen zu zählen, obwohl auch sie nicht weiter zerlegbar sind. Denn die kategorialen Begriffe sollen ja auf die Sinnlichkeit und ihre Formen angewandt werden, damit die Gesetze der Erfahrung umgrenzt werden können.

Obwohl nun dieser zwar unendlich einfache und darum um so bewunderungswürdigere Gedanke Kants die Grundlage einer jeden erkenntnistheoretischen Untersuchung bilden müßte, so ist man bis in die neueste Zeit noch weit entfernt davon, die Bedeutung davon anzuerkennen, geschweige an seine Anwendung zu denken. J)as Bedürfnis jedoch, die kategorialen Begriffe zu gebrauchen, tritt dennoch in manchen philosophischen Schriften klar zutage, wenn auch eine bewußte Verwendung der Kantschen Gedanken dabei fehlt.

Es muß von vornherein zugestanden werden, daß Kant seine Lehre von den Kategorien, so glänzend und weittragend der ihr zugrunde liegende Gedanke ist, nicht nur nicht genügend ausgearbeitet, sondern sie sogar mit großen Fehlern belastet hat. So hat er ihr den ganz unnützen Ballast von dem Schematismus der reinen Vèrstandesbegriffe aufgeladen. Die "Schemata", welche die Anwendung der Kategorien auf die Erfahrungswelt vermitteln und erleichtern sollen, beruhen nach $\mathrm{Kant}$, auf einer verborgenen Kunst in den Tiefen der menschlichen Seele", deren wahre Handgriffe wir der Natur schwerlich je sollen absehen können. Solche Schemata sind nach Kant die Begriffe „Größe", "Zahl", "Grad“ usf. Nun ist es ganz unbegreiflich, wie Kant in so orakelhafter Weise von der Verwendung derartiger Begriffe in der Erfahrung hat reden können. Diese Schemata sind doch nichts weiter, als reine abgeleitete Begriffe, zu deren Auffindung nichts weniger als „eine verborgene Kunst in den Tiefen der. menschlichen Seele" gehört. So ist 
z. B. „Zahl“ das Maß der Zeit, i. e. die Einheit der Größe der Ausdehnung in der Zeit. Der Begriff der Größe selbst aber ist nichts weiter als die Verbindung der Einzelkategorien der Quantität mit dem Begriff „Ausdehnung“, der selbst nichts weiter ist, als eine Teilkategorie der Qualität auf den Raum angewandt. Und so geht es mit allen schematischen Begrifien, sie sind alle auf die ursprünglichen kategorialen Begriffe zurückzuführen, und demnach gänzlich in dem Sinne Kants überflüssig.

Kant war aber auf diesen sonderbaren Schematismus der reinen Verstandesbegriffe ganz offenbar nur darum gekommen, weil seine Kategorientafel nicht richtig aufgestellt war, weil die in ihr enthaltenen Begriffe weder alle reine Verstandesbegriffe, noch auch vollzählig waren.

Was der Anwendung der Lehre Kants außerdem im Wege war, lag darin, daß der Anschauung, dem Gefühl und dessen Gesetzen, deren Auffindung doch das Hauptinteresse einer erkenntnistheoretischen Philosophie sein muß, viel zu wenig in ihr Rechnung getragen ist. Nur auf dem Wege des genauesten Studiums der Kantschen Kritik der reinen Vernunft ist es nachzuweisen, daß Kant den kategorialen Funktionen die Anwendung nicht nur auf das Denken, sondern auch auf die sinnliche Erfahrung ursprünglich zuschrieb, allein diese Meinung des Meisters liegt in seinen Gedankengängen so versteckt, daß sie nur mit Mühe herauszuholen ist. Kant nimmt den Begriff der Erfahrung durchgängig viel zu hoch, sie ist ihm eigentlich identisch mit Wissenschaft.

So kam es, daB Schopenhauer, der mit Recht Kant gegenüber weniger, als ihn ergänzend, die Intellektualität der Anschauung zu beweisen strebte, die Lehre von den Kategorien auf das abfälligste kritisieren konnte, ohne daß ihm auch nur einer seiner so zahlreichen Gegner entgegentrat. Es ist aber sehr merkwürdig, daß der einzige Philosoph, der eine eingehende Kritik der Kantschen Kategorienlehre geschrieben hat, eben Arthur Schopenhauer, in seinem Werke vom Satz des zureichenden Grundes, ohne es zu wollen und zu verstehen, gerade den Beweis von der Richtigkeit dieser Lehre lieferte. Denn seine vierfache Gestaltung des Satzes vom zureichenGrunde ist nichts anderes als die vierfache Art der Kategorie. 
Es erklärt sich aus alle dem, daß die Nachfolger Kants mit seiner allerdings unfertigen und unbrauchbaren Tafel der kategorialen Begriffe die ganze Lehre mit ihrem so einfachen großen und weittragenden Grundgedanken verwarfen.

Erst Albrecht Krause nahm in neuerer Zeit diesen Kantschen Grundgedanken wieder auf. - In seinem großen Werke „Die Gesetze des menschlichen Herzens dargestellt als formale Logik des reinen Gefühls" ') unternahm es dieser Forscher, auf die verlassenen Wege Kants zurückzuweisen, und die verlassene Lehre von den Kategorien neu zu begründen. Er wies, die Gedanken Kants zu Ende denkend, nach, daß die kategorialen Begriffe nicht nur im Gebiet der reinen Vernunft Geltung haben, sondern auf das ganze große Gebiet der Anschauung und der Gefühle gehen, daß es somit möglich erscheint, auf die Kantsche Lehre von den Kategorien eine wirkliche Gefühlswissenschaft zu gründen. Zugleich unternahm er es, die von $\mathrm{K}$ ant aufgestellte Kategorientabelle zu vervollständigen und zu verbessern, so daß sie zur Anwendung auf die sinnliche Erfahrung sowohl, wie auf das reine abstrakte Denken, geeignet wurde.

Wir brauchen für die Behandlung unseres Themas von den Forschungen Albrecht Krauses nur das Wenige, was in den folgenden Seiten enthalten ist, nämlich seine Verbesserung und Vervollständigung der Tabelle der kategorialen Begriffe. -

Albrecht Krause brachte die zwölf Kategorien der Tafel Kants auf sechzehn. Er fügte der Tafel der Quantität die Einzelkategorie "Wenigheit" hinzu, der Tafel der Qualität die Einzelkategorie „Separation“. Noch mehr veränderte er die Tafel der Relation. Diese enthält bei Kant bekanntlich zwei Ioppelkategorien, nämlich Substanz-Akzidenz und Ursache-Wirkung. Es liegt auf der Hand, daß diese Doppelkategorien fallen müssen, denn wie Albrecht Krause nachweist, bilden die Substanzen die Grundlage der Beziehungen und sie beziehen sich aufeinander höchstens als voneinander unabhängig. Die Akzidenzen aber sind die Beziehungen der Ursachen und Wirkungen auf die Substanzen und darum keine

1) $1876 \mathrm{Lahr}$, Schauenburg.

Archiv fll Geschiohte der Philosophie. XXI. 4. 
eigene Kategorie (l. c. p. 132). Auch die zweite Doppelkategorie ist t falsch. Kategorien können nur einfache Begriffe sein, sie können zwar $l^{\circ}$ Wechselbegriffe sein, aber eben darum sind sie ihrem Wesen nach 1 durchaus verschieden. Ursache und Wirkung sind Wechselbegriffe, einer kann ohne den andern nicht sein, aber darum ist eben jeder: ein Begriff für sich.

Die Modalitätstafel Kants ist vollends nicht zu brauchen,, es ist gar nicht $z u$ begreifen, wie Kant den Einzelkategorien 1 Opposita geben und dann diese wieder der Kategorie einverleiben । konnte; wie er dabei so ganz übersah, daß das Oppositum ein aus; zwei Kategorien zusammengesetzter Begriff ist, nämlich aus der einzelnen Modalität und ihrer Negation (Dasein-Nichtsein, Möglich-. keit-Unmöglichkeit, Notwendigkeit-Zufälligkeit).

Die von Albrecht Krause berichtigte Kategorientabelle lautet:

1.

Einheit

Wenigheit

Vielheit

Allheit
2.

Position

Limitation

Separation

Negation
3.

Substanz

Wirkung

Ursache

Wechselwirkung Notwendigkeit

Allein auch an dieser verbesserten Kategorientafel sind noch Korrekturen notwendig, die sich jedoch nur auf die beiden letzten Unterabteilungen. (dynamischen nach $\mathrm{Kan} t$ ) $z u$ erstrecken brauchen.

Wirkung und Ursache sind keine reinen Begriffe. Ursache zerlegt sich von selbst in Ur-Sache, Wirkung aber ist von Werk oder auch von Wirken erst abgeleitet. Ursache ist $z u$ definieren als letzter (oder wenn man lieber will als erster) Grund, der Wechselbegriff von Grund aber ist Folge, und dieser hat den Begriff der Wirkung zu ersetzen. Die beiden Begriffe Grund und Folge aber sind nicht weiter zu zerlegen, wenn man versuchen wollte auch diese zu definieren, würde man auf Tautologien stoßen müssen.

Es gibt sehr viele Vorstellungen, bei denen man von Ursache und Wirkung nicht reden kann, aber es gibt nichts, was nicht Grund oder Folge wäre. In der Mathematik, in der Logik gibt es Grund und Folge. Ursache und Wirkung aber gibt es nur im Gebiet der 
materiellen Veränderung, also innerhalb des Gebietes der Bewegungsvorstellung, diese im weitesten Sinne genommen. Die Veränderung der Materie, deren Form die Bewegung ist, sie erst verlangt Ursache und Wirkung. Diese beiden sind daher zu definieren als Grund und Folge (welche sogenannte aprioristische Begriffe sind) in ihrer Anwendung auf die Veränderungen der Materie. Ursache ist Bewegung als Grund, Wirkung ist Bewegung als Folge.

Mit der Erkenntnis, daß Ursache und Wirkung keine aprioristischen, sondern Erfahrungsbegriffe sind, in denen nur unmittelbar aprioristische stecken, ist auch dem Dilemma ein Ende gemacht, ob das Gesetz der Kausalität eine ursprüngliche, aprioristische Verstandesform, oder ein aus der Erfahrung abstrahiertes Gesetz sei. Offenbar ist das zweite das richtige. Der allgemeine Zusammenhang der Erscheinungen ist nicht geregelt durch das Gesetz der Kausalität, sondern durch den Satz vom zureichenden Grunde, von welchem das erste nur eine Anwendung ist. Das Gebiet seiner Anwendung ist aber nicht nur sehr ausgedehnt, sondern es umfaßt auch gerade die Vorstellungen, die sich zuerst in der Psyche bilden, nämlich die anschaulichen, die Gefühle, also die Naterie und ihre Veränderungen. Die abstrakten Vorstellungen aber, und mit ihnen die Begriffe, entwickeln sich erst auf der von den ersten gelieferten Basis. Was also am spätesten in das Bewußtsein tritt, das sind gerade diejenigen Begriffe, welche die ursprünglichsten Fähigkeiten der Vernunft bezeichnen, das sind eben die kategorialen Funktionen. Wir bilden also den Begriff der Ursache und der Wirkung im Laufe der Erfahrung früher als den des Grundes und der Folge, und daher konnte der täuschende Schein entstehen, als sei das Gesetz der Kausalität das ursprünglichste; denn die Anwendung der Funktionen Grund und Folge auf das sinnliche Material der Anschauung ist eine unmittelbare, die später untersuchende Vernunft hat nur eine einzige Abstraktion zu machen, um auf die kategorialen Begriffe zu kommen.

Können Ursache und Wirkung nicht in der Tabelle der Relation stehen bleiben, so muß auch die letzte Einzelkategorie, die Wechselwirkung, fallen.

Bekanntlich führte Schopenhauer einen heftigen Kampf gegen die Aufstellung dieses Begriffes, den er als eine Art Alarmkanone 
ansieht, zum Zeichen, daß man ins Bodenlose geraten sei. Er hat selbst den richtigen Ersatzausdruck (wenn auch ohne es bestimmt zu beabsichtigen) gefunden, nämlich „Wechselbeziehung“. Entweder ein Ding ist selbständig einem andern gegenüber, i. e. ganz unabhängig in Gefühl oder Betrachtung von einem andern, oder es ist der Grund eines andern, oder seine Folge. Damit sind die Kategorien der Substanz, des Grundes und der Folge gesetzt. Es kann aber ein Ding Grund und Folge einem andern gegenüber zugleich sein, das heißt, es besteht zwischen zwei Dingen eine Beziehung, die jede andere ausschließt, also Wechselbeziehung. Sie findet statt in der Geometrie zwischen räumlichen Bestimmungen, in der Logik zwischen Begriffen, sie wird zur Wechselwirkung in der Anwendung auf materielle Veränderung, wie Anziehung und Abstoßung innerhalb der äußeren Objekte, innerhalb der Empfindung zu Kontrastgefühlen, wie e. g. bei den sogenannten Gegenfarben und vielen andern hierher gehörigen Erscheinungen.

Endlich bedarf auch die Modalitätstabelle Albrecht Krauses noch einer weiteren Verbesserung. Der Ausdruck „Wirklichkeit“ muß fortfallen, da er nichts anderes besagt als „Wirkung“, die als solche erkannt wird. Die Wirklichkeit der Dinge im Gegensatz zu ihrer Scheinbarkeit, besteht eben darin, daß das „wirkliche ${ }^{\text {“ Ding }}$ Wirkungen hat, welche dem scheinbaren fehlen. Noch deutlicher zeigt der Begriff „Notwendigkeit“, daß er kein ursprünglicher, sondern ein durchaus abgeleiteter, zusammengesetzter ist: nämlich das, was die Not wendet.

Es müssen, um die Modalitätstabelle zu vervollständigen, die Wechselbegriffe von "Zufälligkeit“ und von "Möglichkeit“ gesucht werden. Sie müssen sich verhalten wie die Quantitätsbegriffe „eins" und "alles" und "wenig" und "viel“. Der Wechselbegriff von Zufälligkeit ist offenbar die Gewißheit, eine Behauptung, die schwerlich einer eingehenden Begründung bedarf. Der Wechselbegriff der Möglichkeit aber ist die Wahrscheinlichkeit, das was wahr scheint, oder da dieser Ausdruck nicht einfach genug ist, kann man "Anschein“ dafür setzen. Eigentlich sollte man "Scheinlichkeit“ sagen, wenn das ein gebräuchlicher Ausdruck wäre. „Ein Ding ist möglicherweise so" und es ist „dem Anschein nach so" sind Wechsel- 
bestimmungen, nicht in dem Sinne, daß die eine die andere negiert, sondern sie ergänzt, wie die entsprechenden Bestimmungen „viel “ und „wenig,“ wie die kategorialen Begriffe Negation und Position, Separation und Limitation. Wo die Möglichkeit aufhört, beginnt das Gebict der Wahrscheinlichkeit, i. e. des dem Anschein nach Seienden. Die Gegensätze „scheinbar“ und „wirklich“ ergeben sich nicht aus der reinen Anschauung und dem reinen Gefühl, sondern erst aus der Erfahrung an der Hand des Kausalitätsgesetzes, können somit nicht a priori auf Anschauungen gehen (was doch von kategorialen Funktionen verlangt werden muß), sondern verdanken erst der denkenden Analyse ihren Ursprung. Eine anscheinende, wahrscheinliche Vorstellung ist eine solche, die nur durch eine dem Bewußtsein derselben entgegengesetzte sinnliche Erfahrung aufgehoben werden kann. So ist e. g. das Bild einer Rose, welches von einem Hohlspiegel entworfen wird, eine Rose dem Anschein nach, und die Vorstellung, daß eine Rose da sei, kann nur zerstört werden, wenn an derselben Stelle des Raumes, an dem die Rose erscheint, sie nicht auch getastet wird.

Die verbesserte Modalitätstabelle muß demnach lauten: Zufälligkeit, Möglichkeit, Anscheinlichkeit (wofür besser, weil dieser Ausdruck ungebräuchlich ist, Wahrscheinlichkeit zu setzen ist), Gewißheit.

Die gesamte verbesserte Kategorientabelle, wenn man die Einzelkategorien so ordnet, daß innerhalb einer Reihe immer ein Fortschritt vom Kleineren zum Größeren statt hat; i. e. die Begriffssphäre immer vom Einzelnen zum Allgemeineren erweitert wird, ist nunmehr die folgende:

$\begin{array}{llll}\text { Quantität } & \text { Qualität } & \text { Relation } & \text { Modalität } \\ \text { Einheit } & \text { Position } & \text { Substanz } & \text { Zufälligkeit } \\ \text { Wenigheit } & \text { Limitation } & \text { Folge } & \text { Möglichkeit } \\ \text { Vielheit } & \text { Separation } & \text { Grund } & \text { Wahrscheinlichkeit } \\ \text { Allheit } & \text { Negation } & \text { Wechselbeziehung } & \text { Gewißheit }\end{array}$

Auf diese sechzehn kategorialen Begriffe, welche ebensovielen transzendenten Vermögen der Psyche entsprechen (welche forderbar sind und innerhalb der Vernunft eben als Begriffe erscheinen), und ihre Anwendung auf die Formen der Sinnlichkeit (Raum, Zeit, 
Empfindung, Bewegung) müssen sich alle aus der Erfahrung abziehbaren Begriffe zurückführen lassen. Es ist dabei vorausgesetzt, daß die 'Tafel richtig konstruiert ist, ist diese Voraussetzung falsch, so muß sie korrigiert werden, der ihr zugrunde liegende Gedanke jedoch verliert dadurch nichts an seiner Bedeutung.

Albrecht Krause hat in seinem großen Werke gezeigt, welche Begriffe entstehen, wenn man die kategorialen Begriffe auf die Formen der Sinnlichkeit anwendet, und eine große Anzahl abgeleiteter, einfach und vielfach kombinierter Begriffe aufgestellt, welche im Einzelnen zwar der Verbesserung bedürftig sind, aber den Beweis liefern, daß in einem jeden Begriffe, der eine Anschauung oder ein Gefühl ausdrückt, die kategorialen Funktionen stecken (in Verbindung mit den verschiedenen Sinnlichkeitsradikalen), welche Kant zunächst nur für das reine Denken aufgestellt hat. Kant hat den Begriff Erfahrung immer viel zu hoch aufgefaßt und genau genommen mit Wissenschaft identifiziert. Albrecht Krause hat daher das große Verdienst, die Lehre Kants bedeutsam erweitert und gezeigt zu haben, daß dessen grundlegende Gedanken im Gebiete der rein sinnlichen Erfahrung dieselbe Macht haben wie innerhalb der reinen Vernunft.

Es ist nun zu untersuchen, welche kategorialen Begriffe mit dem Begriff der Freiheit, dessen Definition wir suchen, in Verbindung zu bringen seien.

Freiheit ist ein relativer Begriff, da man bei seiner Bestimmung im einzelnen immer zu fragen hat: Freiheit wovon? und somit ist klar, daß es sich auch bei der allgemeinen Definition nur um die sogenannten dynamischen Kategorien handeln kann, nämlich die der Relation und die der Modalität.

Die Relation schließt in sich die Kategorien der Substanz, der Folge, des Grundes und der Wechselbeziehung. Diese Reihe ist somit ein anderer, und zwar der ursprüngliche, Ausdruck des Satzes vom zureichenden Grunde, von welchem, wie wir gesehen haben, das Gesetz der Kausalität nur ein besonderer Fall ist. Grund und Folge angewandt auf materielle Veränderung, also auf Bewegung, ergeben die unmittelbar abgeleiteten Begriffe der Ursache und Wirkung. 
Diese Kategorienreihe kann mit dem Begriffe der Freiheit nichts zu tun haben, da sie eigentlich das Gegenteil derselben ausdrückt, die strenge Gesetzmäßigkeit.

Es bleiben also zur Bestimmung des Freiheitsbegriffes nur noch die Kategorien der Modalität übrig.

Und es leuchtet auf den ersten Blick ein, daß diese Kategorienreihe geradezu der Ausdruck, und zwar der ursprünglichste Ausdruck der Freiheit ist.

Zufälligkeit bedeutet die vollkommene Unabhängigkeit einer Erscheinung, sei sie rein anschaulicher, sei sie abstrakter Natur, vom Gesetz der Kausalität im engeren, vom Satz des zureichenden Grundes im weiteren Sinn. Eine Erscheinung, sei sie äußeres räumliches Objekt, sei sie seelische Empfindung oder abstrakter Gedanke, wird, wenn sie zufällig ist, schlechthin als Existenz aufgefaßt, sie ist niemals Ursache oder Wirkung, niemals Grund oder Folge.

Möglichkeit bedeutet die Auffassung einer Erscheinung als einer Wirkung oder einer Folge, deren Ursache oder deren Grund zwar als existierend vorgestellt oder denkend vorausgesetzt wird, aber unbestimmt bleibt.

Umgekehrt bedeutet Wahrscheinlichkeit die Auffassung einer Erscheinung als Ursache oder Grund, deren Wirkung oder Folge zwar als existierend vorausgesetzt oder gedacht wird, aber nicht mit Bestimmtheit in das Bewußtsein erhoben zu werden braucht.

Gewißheit endlich bedeutet die Auffassung der Erscheinung als gegebene Wirkung oder Folge aus gegebener Ursache oder gegebenem Grunde.

I)a der Satz vom zureichenden Grunde die Art unserer Erkenntnis darstellt, und dieser Satz ausgedrückt ist in den Kategorien der. Relation, so drücken die Kategorien der Modalität die Art aus, in der dieser Satz angewandt wird. Die Kategorie der Zufälligkeit befähigt uns von seiner Anwendung überhaupt abzusehen, die Erscheinungen ohne jede Beziehung aufeinander, als Substanzen in ihrer Selbständigkeit aufzufassen, während die Kategarie der Gewißheit uns befähigt, das Gesetz in seiner ganzen Strenge anzuwenden. Die beiden übrigen Kategorien gestatten unserem Vorstellungs- und 
Denkvermögen eine Einschränkung (oder eine Ausdehnung, wie man will) nach der einen oder der andern Seite.

Wir wären somit zu der gesuchten Definition des Begriffes „Freiheit" gelangt. Freiheit ist Unabhängigkeit vom Gesetz des zureichenden Grundes im allgemeinen, vom Gesetz der Kausalität im besondern Sinn. Die Art und die verschiedenen Grade dieser Unabhängigkeit sind ausgedrückt in den kategorialen (also nicht weiter reduzierbaren, ursprünglichen oder aprioristischen) Begriffen der Modalität. Man kann diese Kategorien mit einem Ausdruck Kants, der freilich in einem ganzen andern Zusammenhang in der Kritik der praktischen Vernunft gebraucht ist, ihn auf die Kritik der reinen Vernunft (wohin er wirklich gehört) übertragend, geradezu als die Kategorien der Freiheit bezeichnen.

Wir gelangen hiermit zu unserem eigentlichen Thema.

\section{Zweites Kapitel.}

\section{Die Willensfreiheit.}

Das uns allen wohlbekannte Gefühl, welches durch den Begriff „Freiheit" sprachlich ausgedrückt wird, beruht also, wie im vorhergenden auseinandergesetzt ist, auf der Unabhängigkeit vom Satze des zureichenden Grundes im allgemeinen, von dem Gesetze der Kausalität im besonderen, philosophisch ausgedrückt auf den seelischen Mächten der Modalitätskategorien. Alle Erscheinungen, welche die Anwendung dieser Kategorien im Gefühl, in der Anschaung, in der vernunftgemäßen Betrachtung (der denkenden Analysis) zulassen, können daher das Prädikat der Freiheit erhalten. Sie bekommen dasselbe beigelegt dadurch, daß sie zufällig, möglich, wahrscheinlich sein können.

Dies gilt von allen Naturerscheinungen, auch von denen die wir als objektive zu bezeichnen gewohnt sind. Wenn wir beispielsweise sagen: „es kann ein Gewitter kommen" oder "es gibt wahrscheinlich ein Gewitter", so heißt das in unsere Ausdrucksweise übersetzt: es steht dem Gewitter frei zu kommen, denn soviel wir sehen, ist der Zustand der Atmosphäre derart, daß darin keine Ursache liegt, welche sein Kommen verhindern würde. Dabei geben wir uns keine Mühe, diesen Ursachen genauer nachzugehen, 
obgleich wir uns bei konsequentem Nachdenken sagen müssen, daß das Gewitter kommen oder ausbleiben muß, weil es von dem Zustande der Atmosphäre in der bestimmtesten Art abhängt. Wir sagen: es fällt mir zufällig ein Stein auf den Kopf, also kümmern wir uns nicht um die Ursachen dieser Wirkung, obgleich es doch ganz sicher ist, da $B$ diese Erscheinung aus dem Zusammentreffen zweier Kausalketten abgeleitet werden kann, um die wir uns aber bei der Auffassung jener Erscheinung nicht kümmern, und uns folglich dabei von der Kausalkette unabhängig fühlen.

Was von den Naturereignissen gilt, das gilt auch von unseren Handlungen, welche die räumlichen Erscheinungen unseres Willens in der Form der Bewegung sind. Wir handeln dann frei, wenn wir das tun können, was wir wollen, das heißt, wenn kein Grund oder keine Ursache in unserem Bewußtsein sich finden läßt, weder in uns selbst noch in den Objekten, welche einen hindernden Einfluß auszuüben imstande wären. Wenn ich zufällig etwas tue, wenn es mir möglich ist, etwas $z u$ tun, wenn ich wahrscheinlich eine Handlung ausfübren werde, dann tue ich das entweder ohne jede Berücksichtigung von Gründen oder Folgen, Ursachen oder Wirkungen (also zufällig) oder mit einer unvollständigen Berücksichtigung derselben (also möglicher- resp. wahrșcheinlicherweise), das heißt mit größerer oder geringerer Freiheit. Eine denkende Analyse meiner Handlungen wird mir gleichwohl jedesmal das Resultat ergeben müssen, daß diese ohne Grund und Folge, oder ohne Ursache und Wirkung gar nicht in die Erscheinung treten können, daß sie immer streng determiniert sind, nur in meiner Betrachtungsart und meiner Gefühlsweise dies nicht regelmäßig sind.

Was von den Handlungen gilt, die doch nichts sind als die Wirkungen des Wollens (genauer des Entschlusses, der kategorial definiert werden muß als Wollen mit Gewißheit funktioniert) in räumlicher Erscheinung, muß ganz genau so von allen Willenserscheinungen gelten, seien sie, als Begriffe ausgedrückt, einfach oder mehrfach funktioniert, sei also e. g. eine Willenserscheinung eine „Regung" oder eine "Laune" (Wille als Zufälligkeit), oder Neigung resp. Absicht (Wille als Möglichkeit), oder "Lust" (Wille als Wahrscheinlichkeit). Gleichviel was ich mag, begehre, wünsche, beabsich- 
tige oder wozu ich mich entschließe, bei einer genauen denkenden Analyse muß sich immer zeigen, daß für mein Wollen ein bestimmter Grund, oder eine, in oder außer mir liegende, Ursache existiert. Aber wenn ich aus Laune, also zufällig handle, kümmere ich mich in meinem Bewußtsein nicht um Grund oder Ursache dieser Laune, meine Handlung erscheint mir, und unter bestimmten Bedingungen auch Andern, „frei“ zu erfolgen. Neige ich zu einer Handlung, so ist sie möglich, wenn ich ihre Folgen nicht kenne, oder nicht kennen will. Gleichwohl ist es klar, daß ich die Handlung ausführen werde oder nicht, je nachdem die Gründe oder Ursachen für das eine oder das andere in oder außer mir vorhanden sind, oder fehlen. Treibt es mich zu einer Handlung, so ist sie wahrscheinlich, das will sagen, es ist Grund dafür vorhanden, aber dieser Grund kann in meinem Bewußtsein durch einen Gegengrund aufgehoben werden, Gleichwohl ist es bei der denkenden Analyse gewiß, daß die Handlung entweder ausgeführt wird oder nicht, je nachdem der Gegengrund existiert oder nicht.

1)ie Freiheit des Handelns ist somit nur ein besonderer Fall der Freiheit des Wollens im allgemeinen. Eigentlich identisch damit ist die so viel behandelte Freiheit des Wählens. Die sogenannte freie Wahl besteht in nichts anderem, als daß von zwei Handlungen die eine oder die andere möglicherweise, oder auch wahrscheinlicherweise, ausgeführt wird. Wenn von zwei sich einander ausschließenden Handlungen die eine gewählt wird, so trägt in dem Streit zweier sich entgegenstehender Willensrichtungen die stärkere immer den Sieg davon. Man sagt (Schopenhauer), daß wir die Wahl zwischen zwei Motiven haben. Allein was man "Motiv" nennt, ist nichts weiter als „Wille als Grund“, i. e. „Beweggrund". Zwei Motive (auch identisch mit Zweckbegriff) sind elnfach zwei verschiedene Wollungen, die in unserer Psyche miteinander kämpfen. Solange der Kampf nicht entschieden ist, erscheint es im Gefühl oder auch in der untersuchenden Vernunft möglich oder wahrscheinlich, daß sowohl das eine wie das andere Motiv den Sieg davontrage. Gleichwohl ist es sicher, daß immer nur das stärkere Motiv siegt, es beruht also die Freiheit der Wahl lediglich auf der Unkenntnis der Stärke der Motive, und nur diese Unkenntnis 
bedingt die Anwendung der Modalitätskategorien, welche im andern Falle denen der Relation das Feld zu räumen hätten.

Ebenso wie jedes Wollen in seinem Verlauf abhängig ist vom Gesetz des zureichenden Grundes, so ist es auch sein Entstehen, seine Existenz. Die Frage: ist irgend eine Willensexistenz frei zu entstehen? ist zu verneinen, denn eine jede Willensregung hat einen Grund in einem andern Wollen, der im einzelnen wohl schwer aufzufinden oder ganz verborgen bleiben kann, aber dennoch da sein muß, oder man müßte die Annahme machen, daß es Erscheinungen gebe, die keinen Grund oder im engeren Sinne keine Ursache hätten. Einer solchen ungereimten Annahme, die gleichwohl, um die Willensfreiheit in objektivem Sinne zu retten, gemacht worden ist, steht eben das Gesetz vom zureichenden Grunde, welches als das allgemeine die innere Erscheinung und als das spezialisierte der Kausalität die äußere Erscheinung beherrscht, entgegen, welche beide ihren gemeinsamen Ansdruck finden in der Lehre vom psychophysischen Parallelismus. Diese Lehre wird in neuester Zeit Fech ner zugeschrieben, der sie jedoch sich erst in zweiter Linie zu eigen gemacht hat. In ihrer ausgeprägten Form stammt sie vielmehr von Arthur Schopenhauer. Sie bildet die Basis seiner gesamten Weltanschauung in erkenntnistheoretischer Beziehung. In ihren Anfängen ist sie wohl auf Spinoza (denkende und ausgedehnte Substanz) zurückzuführen.

Während die Form unseres geistigen Wesens die Zeit ist, erscheint es dennoch auch räumlich (auch das der Tiere) als Sinnesorgane und Gehirn mit dem sensiblen und dem motorischen Nervensystem. Ein jeder Willensakt, soweit er bewußt ist (unbewußte Vorgänge gehen uns hier nichts an), ist in seiner räumlichen Erscheinung ein Vorgang in der Hirnrinde mit daran sich anschließenden elektrochemischen Vorgängen im zentralen und peripherischen Nervensystem. Wäre das Gehirn des Menschen durchsichtig zu machen, so würden wir während seines ganzen Lebens die Bewegungen in der Hirn- und Nervensubstanz wahrnehmen können (ja man vermöchte die seines eigenen Hirns im Spiegel zu beobachten), welche doch sämtlich in andern Bewegungen ihre Ursache haben, nämlich in dem, was die Physiologen als "Sinnesreize" bezeichnen, 
ein Ausdruck, der freilich falsch ist, denn ein Sinnesreiz ist schon wirkliche Empfindung, man sollte statt dessen sagen „äußere“ (außerhalb des Organismus liegende) Ursachen. Es geht also der Ablauf des ganzen Lebens mit seinen sämtlichen Willenserscheinungen, welche die Form der Bewegung haben, durchaus streng nach dem Gesetze der Kausalität vor sich, und somit ist eine jede Freiheit im Sinne der Indeterministen völlig ausgeschlossen.

Das aber, was in der räumlichen (äußern, objektiven) Erscheinung Ursache und Wirkung ist, das ist in der zeitlichen (inneren, subjektiven) Grund und Folge. Es existieren hier nicht zwei voncinander im Grunde ihres Wesens verschiedene Dinge, sondern ein und dasselbe, nur von verschiedenen Seiten betrachtet. Was innerlich Wille ist, das ist äußerlich Bewegung im Raum (gegenüber der Empfindung in der Zeit), und umgekehrt. Wäre also der Wille im Sinne des Indeterminismus frei, so müßte den Willensvorgängen im Gehirn und dem Nervensystem, die sie doch nur objektivieren, Bewegungen entsprechen, die keine Ursache hätten, das aber ist undenkbar. Daß jedoch in der räumlichen Erscheinung alles streng necessitiert sei, dagegen in der seelischen, zeitlichen, ein ursachloses Geschehen vorkomme, ist geradezu heller Widersinn, obgleich es noch vor nicht langer Zeit von juristisch-philosophischer Seite behauptet worden ist. Denn Ursache und Wirkung sind nichts anderes als die äußeren Erscheinungen von Grund und Folge, man müßte also behaupten können, daß es irgend etwas gebe, was als Existenz keinen Grund hätte, grundlos wäre.

Iie Art der Willensfreiheit, die praktisch am wertvollsten ist, nämlich die Möglichkeit das zu tun, was man will (wobei die Modalitätsbestimmung auf äußere Ursachen geht, von denen der Wille unabhängig sein muß, während es sich bei der Wahlfreiheit und auch bei der moralischen Freiheit um innere Ursachen oder innere Gründe handelt), diese ist bei uns in Deutschland von jeher nicht leicht $z u$ finden gewesen, und deshalb suchen sich Juristen und philosophierende Theologen eine transzendente Freiheit, statt einer immanenten, zu konstruieren. Selbst Arthur Schopenhauer verfiel in eine große Petitio principii, wenn er der Meinung war, die Freiheit müsse im Esse liegen, da sie nicht im Operari zu finden 
sei, aus dem er sie unwiderleglich vertrieben hatte. Denn woher wußte er und sein Lehrer Kant so sicher, daß die Freiheit überhaupt irgendwo liegen müsse?

Die Freiheit, wie sie Kant und Schopenhauer verstanden wissen wollten, liegt, wie sie beide gefunden haben, nicht im Operari, und auch im Esse kann sie nicht liegen, soweit unsere Vernunft sich davon Rechenschaft zu geben vermag, was im letzten Kapitel dieser Abbandlung gezeigt werden soll.

Freiheit liegt einzig und allein in unserm Gefühl.

Dies Gefühl besteht in dem Bewußtsein, daß eine Erscheinung nicht streng necessitiert sei. Freiheit des Wollens bedeutet also nichts anderes, als daß ein Wollen nicht mit Gewißheit habe entstehen-oder verlaufen müssen, sondern daß es nur wahrscheinlich, oder nur möglich und zufällig sein könne. Es verhält sich mit den Äußerungen des Willens innerhalb der menschlichen Seele, also mit den inneren Ereignissen, nicht im mindesten anders als mit jedem beliebigen äußern Naturereignis, das sich in unserer Erfahrung findet. Nehmen wir das alte Beispiel vom Gewitter. Es kommt „zufällig“, wenn uns die Zustände der Atmosphäre ganz und gar nicht in das Bewußtsein kommen, wir merkten aus irgend einem Grunde nicht darauf. „Es ist möglich, daß ein Gewitter kommt", sagen wir in dem Falle, daß etwa die Schwüle der Luft in uns diese Vorstellung entstehen läßt; „es kommt wahrscheinlich ein Gewitter" sagen wir, wenn wir Wolken von bestimmter Beschaffenheit sich sammeln sehen. In allen diesen Fällen liegt aber in den Verhältnissen der Atmosphäre, in derWindrichtung etc., sicher die Ursache, ob das Gewitter kommt oder nicht, ob es entsteht oder, bereits da, sich dennoch wieder verzieht. Wären wir imstande gewesen, die Ursachen zu finden oder auch nur zu suchen (wovon uns irgend ein Motiv, sei es e. g. Denkfaulheit oder eine uns lebhafter anziehende Vorstellung, abzog), so konnte von mögen oder wahrscheinlich sein nicht gesprochen werden. Das Gewitter konnte kommen, es konnte aber auch wegbleiben, es war somit in unserm Sinne frei, in unserm Gefühl frei, zu kommen oder zu gehén - weil wir seinen Ursachen nicht nachforschen wollten. 
Nicht ein Jota anders verhalten sich die innerhalb der Psyche auftretenden Ereignisse, die wir als Erscheinungen des Willens kennen. Mögen sie Regungen oder Neigungen und Triebe, mögen sie Begehrung oder Verwerfung darstellen, seien sie charakterisiert als Laune, als Wunsch oder als EntschluB, immer haben sie eine ganz bestimmte Entstehungsursache oder bestimmten Entstehungsgrund, mag dieser sich auf eine unmittelbar erkannte Sinneswahrnehmung zurückführen lassen, oder auf eine Gedankenreihe, oder endlich in den Tiefen des Allgemeingefühls verborgen bleiben. Die Analyse mittels der denkenden Vernunft lehrt dies für alle Fälle ohne Ausnahme, während wir im einzelnen den Grund der Entstehung einer Willenserscheinung nicht nur uicht kennen, sondern ihn auch gar nicht $z u$ erforschen versucht sind, in andern Fällen ihn mehr oder weniger genau kennen, ihu mehr oder weniger zu erforschen neigen. Streng genommen wissen wir beim Nachdenken darüber sehr wohl, daß ein jedes Wollen genau necessitiert ist, dennoch sind wir imstande, uns, ohne uns um Grund und Folge, Ursache und Wirkung zu kümmern, einfach unsern Gefühlen zu überlassen. Ja es gibt nur verhältnismäßig wenige Menschen, denen jenes Wissen eigen ist, daß alle Erscheinungen streng necessitiert sind, das Leben der meisten Individuen ist ausgefüllt durch Anschauen und Fühlen, das der wenigsten auch nur zum großen Teil durch bewaßtes Erkennen. Aber selbst das bewußte Erkennen ist immer von dem Gefühl begleitet. Dies erklärt sich durch seine Natur, Erkennen ist nur eine besondere Art des Wollens, selbst als vernünftiges Denken. Die Gefühle des Zufälligen, Möglichen, Wahrscheinlichen und Gewissen begleiten nicht nur das Fühlen im engeren Sinne (Lust- und Unlust), nicht nur die unmittelbare sinnliche Anschauung, sie sind ebenso unzertrennlich von den langen Gedankenreihen der wissenschaftlichen Forschung, sei es Experimentalarbeit, sei es Mathematik oder reine Logik. Das Gefühl der Freiheit tritt in allen diesen Gebieten, so verschieden sie voneinander sind, auf. Im Tun sowohl wie im Denken können wir uns frei fühlen. Wir sind in der Tat frei, aber wovon? Von nichts weiter als von der bewußten Anwendung des Kausalgesetzes und, allgemeiner gesagt, vom Gesetz des zureichenden Grundes. 